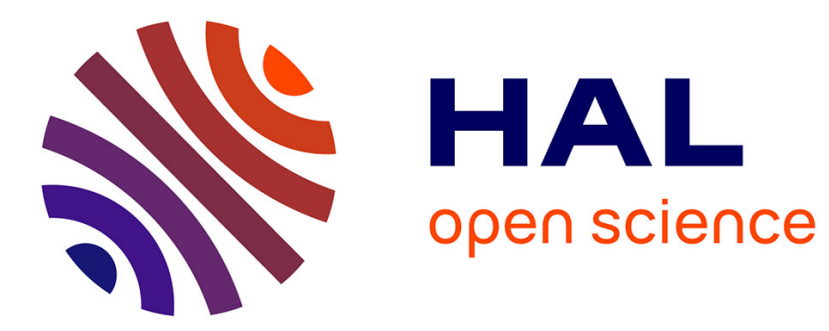

\title{
Le mausolée de Marcus Rufius Catullus, curateur des Nautes du Rhône, à Gélignieux (Ain) - CIL XIII, 2494
}

\author{
André A. Buisson
}

\section{To cite this version:}

André A. Buisson. Le mausolée de Marcus Rufius Catullus, curateur des Nautes du Rhône, à Gélignieux (Ain) - CIL XIII, 2494. Revue archéologique de Narbonnaise, 1991, pp. 141-158. hal-02447124

\section{HAL Id: hal-02447124 \\ https://hal.science/hal-02447124}

Submitted on 27 Jan 2020

HAL is a multi-disciplinary open access archive for the deposit and dissemination of scientific research documents, whether they are published or not. The documents may come from teaching and research institutions in France or abroad, or from public or private research centers.
L'archive ouverte pluridisciplinaire HAL, est destinée au dépôt et à la diffusion de documents scientifiques de niveau recherche, publiés ou non, émanant des établissements d'enseignement et de recherche français ou étrangers, des laboratoires publics ou privés. 


\title{
LE MAUSOLÉE DE MARCUS RUFIUS CATULLUS, CURATEUR DES NAUTES DU RHÔNE À GÉLIGNIEUX (Ain) CIL XIII 2494
}

\author{
André BUISSON
}

«Il est donc sage de donner cette inscription comme un simple renseignement et d'engager les habitants de l'Ain à signaler ce monument si le hasard ou les recherches le mettent à nouveau en lumière ${ }^{(1)}$ ».

L'attention du chercheur s'intéressant aux inscriptions funéraires des bords du Rhône en amont de Lugdunum est immanquablement attirée par l'inscription CIL XIII 2494, découverte à Gélignieux (Ain).

Il s'agit d'un tombeau de caractère monumental, détruit vers 1705 et connu outre son inscription rapportée par l'auteur du CIL XIII, par un dessin de l'Abbé Claude de Veyle (2), rapporté de mémoire d'après les indications des personnes ayant quinze ans plus tôt participé à la démolițion de l'édifice, considéré par le curé du lieu comme un lieu de débauche notoire (sic).

Du monument ne subsistent aujourd'hui que deux fragments de l'inscription et un cippe-autel sans doute encore à sa place d'origine, le long du chemin de desserte menant de la route départementale à la rive du fleuve.

Dans l'historiographie régionale, le monument a laissé chez les chercheurs des souvenirs divers, voire dubitatifs: forme de l'inscription et localisation du tombeau ont été débattues jusqu'à notre siècle. Deux sites géographiques ont ainsi été concurrement défendus par les historiens du département de l'Ain :

- Les Marches, commune de Saint-Benoît, à la suite des assertions de Guichenon (3),

(1) Comte DE MOYRIA-Mall.LA. Momuments romuins du département de l'Ain. expliqués par..., Bourg, 1836, p. 77.

(2) Claude DE VEYLE, Explication des Antiquiris romaines qui se trou'ent dans les pays de Bresse, Bugey, Valromey et Gex, manuscrit. vers 1720, édité part A. Buisson. Bourg-s’n-Bresse. 1985. collection des Sources de l'Histoire de l'Ain.

(3) Par exemple J. Havisezo. Les wies antiqués é romaines du départemen de l'Ain. Bourg, 1917. p. 22 : «M. Philippon indique par erreur ce tombeau comme ayant été découverı à Gélignieu»: M. de Moyria reste, comme nous l'avons vu plus haut, beaucoup plus circonspect. 
- Gélignieux, peut-être à la suite de Claude de Veyle. A. Carena, écrivant vers 1740, rapporte : «à Belissieu, paroisse près du Rhône, diocèse de Belley, auprès du château de Murs, il y a au milieu d'un pré une sépulture toute bâtie... et en dos d'âne que les villageois appellent «la Garde»(4).

\section{L'EMPLACEMENT DU TOMBEAU}

Le tombeau dans son enclos funéraire avait été érigé sur la pointe occidentale de la petite plateforme alluviale dominant le cours du Rhône, à une altitude de $222,50 \mathrm{~m}$ et dominant le cours du fleuve d'une dizaine de mètres environ ${ }^{(5)}$. La plateforme alluviale s'amenuise à cet endroit pour ne plus conserver qu'une largeur d'une soixantaine de mètres, entre fleuve et montagne (fig. 1). Le crippe-autel se trouve sur la limite de la parcelle A 116, le long du chemin rural.

Le tombeau occupait une surface que l'on peut évaluer à $15 \mathrm{~m}^{2}$. Nous ne possédons que peu d'indications "modernes" sur son emplacement, mais le cippe-autel anépigraphe dont nous avons déjà parlé peut aider à la localisation de l'ensemble funéraire. Derrière l'église, se trouve aujourd'hui un jardin dont une partie est ensemencée en prairie, et au centre de ce pré, à une quinzaine de mètres de la limite de la parcelle, on remarque un espace dont la végétation tranche sur le pré environnant : seules des ronces et orties poussent sur un espace inférieur à $20 \mathrm{~m}^{2}$; lorsque l'on sait que ce type de végétaux affectionne les terrains rocailleux, on peut faire le parallèle avec la présence à faible profondeur d'un socle qui pourrait être le massif de fondation du tombeau de Marcus Rufius Catullus. Cet espace se distingue sur les photographies aériennes en fausses couleurs.

Pour arriver là où on les a localisées aujourd'hui, les pierres ont suivi un curieux parcours : le mausolée fut démoli vers 1700-1705. Ses matériaux furent remployés au pavage de l'église d'alors, qui se trouvait au lieu-dit «La chapelle», en haut du village actuel, dans un emplacement rendu insalubre par la présence de sources nombreuses et abondantes. Soixante quinze ans plus tard, cette même église fut démolie sur le vœu des habitants de la paroisse et remplacée par l'église actuelle, dont la construction fut achevée en $1785^{\prime \prime \prime}$. Curieux dessein du sort, ce dernier dérangement rapportait les pierres à quelques dizaines de mètres de l'emplacement du tombeau antique.

\section{L'INSCRIPTION}

Nous sommes tributaires de la lecture et de l'interprétation qu'en a données Guichenon (fig. 2a: le texte d'après Samuel Guichenon : fig̣. $2 b$ : le texte d'après de Veyle). Tous les auteurs postérieurs ont suivi sa division du texte en deux parties. l'une sur un support de forme triangulaire (cf. par exemple O. Hirschfeld. dans notice au CIL XIII, v.1-5: in aetoma; nous n'avons pu ni confirmer ni infirmer cette hypothìse. le fragment 3a. étant martelé et sans doute recoupé à un gabarit différent de l'original), l'autre sur un support en forme de bandeau (7) (cf. la figure $3 \mathrm{~b}$; dimensions: $0,80 \times 0,60 \times 0.45 \mathrm{~m}$. dimensions der lettres: $0.05 \mathrm{~m} ; \varnothing$ des $0: 0,055 \mathrm{~m}$ ).

(4) N.v., cité par Hirschlcild dans sa nolk: al: $(\% 11$. XIll 2494

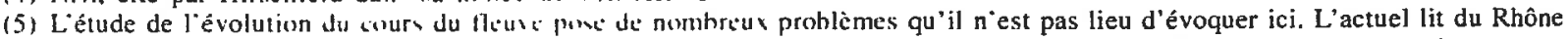

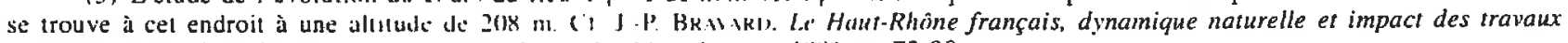

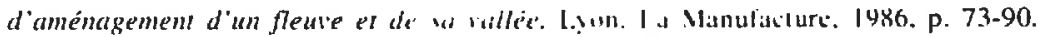

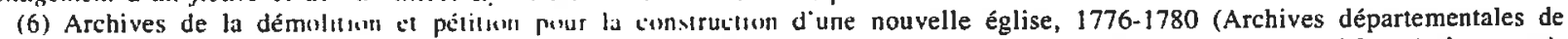
l'Ain, cote (300); lettre du comte de Somont du $\mathrm{I}$ : uptembre 1780 désireux de rapprocher l'église du château de Murs (même cote).

(7) Champ épigraphique : tung: $0.60 \mathrm{~m}: 1.4 \mathrm{~s}: 11.38 \mathrm{~m}:$ hautcur des lettres: $0,05 \mathrm{~m}$; diam. des $\mathrm{O}: 0,055 \mathrm{~m}$. La partie inférieure de l'inscription devait donc être vuppurtće par un lintèu lormé de trois blocs de dimensions sensiblement voisines, soit une longueur d'environ $2.40 \mathrm{~m}$ pour une largeur de $0.60 \mathrm{~m}$ s't un thamp épigraphique d'environ $2,00 \mathrm{~m}$ par $0,38 \mathrm{~m}$. 


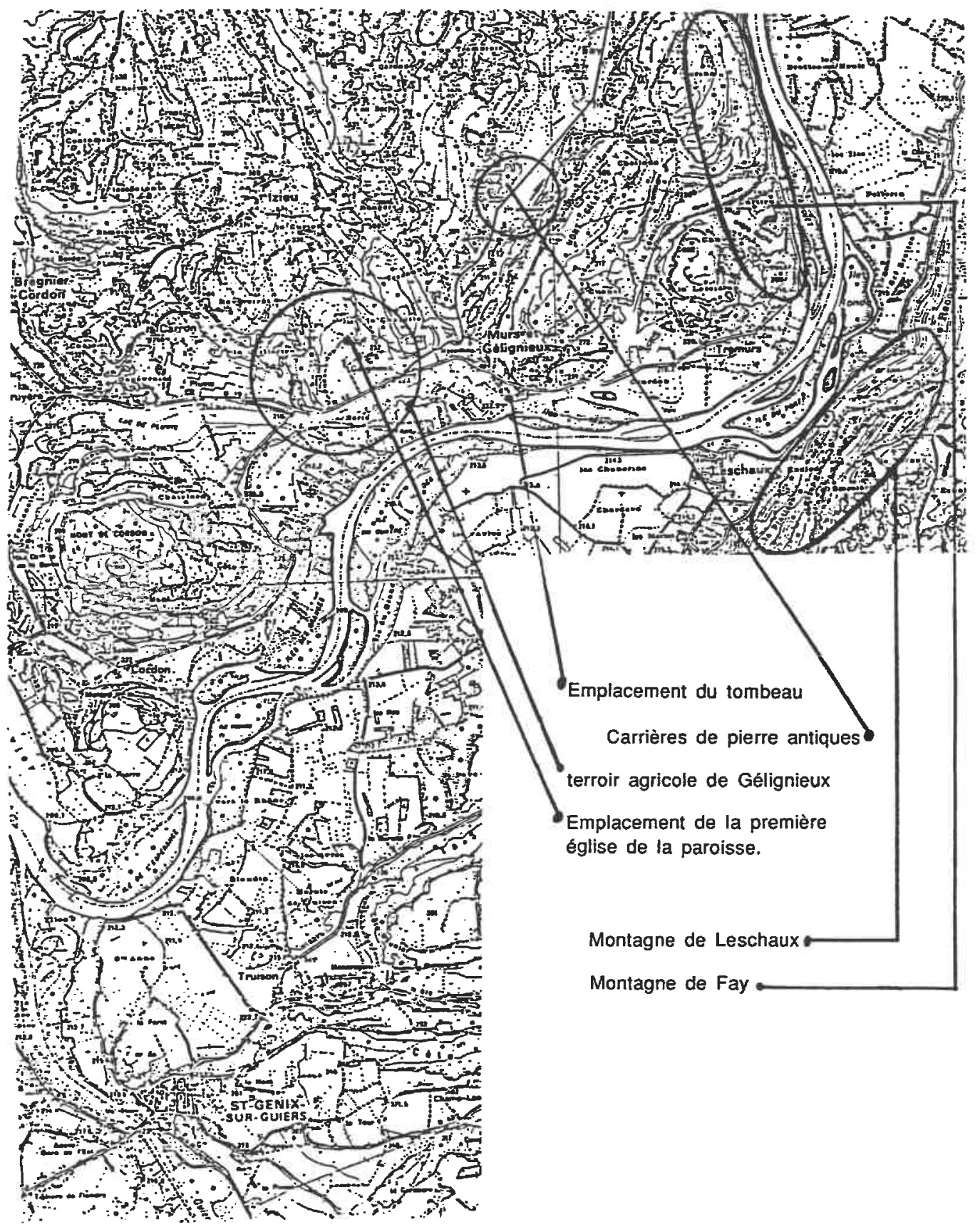

Fig. 1. - Le site antique dans son terroir. 


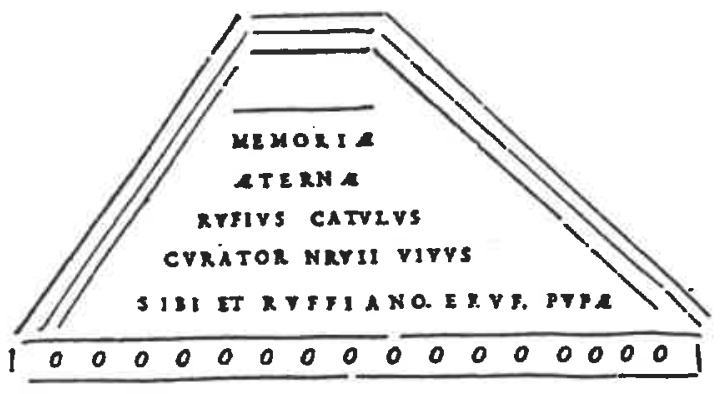

ET RVIA SAGIRIATA FIL DEFTNCTE ANNOR XXIL

EDICIAM CVMVINEA. ET MVRIS AD OPYS

CONSVMMANDYM ET TYTELAM EIYSET AD AD CENAM

OMNISY RI CONTIS FONENDAM XL THN PERET.

SICVTI T RVDECAX 2 O CONSTHATYR HOC ORYS

SV A SCIA EST

$H A C$ O. S. L H N. S.
Fig. 2a. - L'inscription de Gélignieux d'après le dessin de S. Guichenon, Histoire de Bresse et de Bugey, t. 1, p. 10-11 : «aux Marches, sur une pierre de taille d'onze pieds de quarrure».

Partie supérieure :

MEMORIAE • AETER

NAE $\cdot M \cdot$ RVFIVS $\cdot$ CATVL

LVS $\cdot$ CVRATOR $\cdot \mathrm{N} \cdot \mathrm{RH}$

VIVVS $\cdot$ SIBI $\cdot$ ET $\cdot$ RVF $\cdot$ RVFIANO $\cdot \mathrm{F}$

RVF • PVPAE - FIL

Partie inférieure :

ET - RVFIAE - SACIRATAE - FIL - DEFVNCTAE - ANNOR

XXII - AEDICLAM - CVM - VINEA - ET - MVRIS - AD - OPVS

CONSVMMANDVM $\cdot$ ET $\cdot$ TVTELAM $\cdot$ EIVS $・$ ET $・$ AD $・$ CENAM $・$ OMNI

BVS • TRICONTIS • PONENDAM - XII ・ IN ・ PERPET ・ SICVT • PETRVDECA

METO $\cdot$ CONSVMATVR $\cdot$ HOC $・$ OPVS $\cdot \mathrm{SVB} \cdot \mathrm{ASCIA} \cdot \mathrm{EST} \mathrm{HAEC} \cdot \mathrm{O} \cdot \mathrm{S} \cdot \mathrm{L} \cdot \mathrm{H} \cdot \mathrm{N} \cdot \mathrm{S}$

Memoriae acterlnae M(arcus) Rufius Catullus curator n(autarum) rh(odanicorum) I uiuus sibi et Ruf(io) Rufiano f(ilio) | Ru(fiae) Pupae fil(iae) ||

et Rufiae Saciratae fill(iae) defunctae annor(um)(XXII, aedic(u)lam cum uinea et muris ad opuslconsummandum et tutelam eius et ad cenam omnibus tricontis ponendam denariis II in perpet(ua) sicut petrudecalmeto comsumatur; hoc opus sub ascia est haec o(pera) s(iue) l(ocus) h(eredem) n(on) s(equentur).

A la mémoire éternelle. Marcus Rufius Catullus, curateur des Nautes du Rhône a, de son vivant, pour lui-même et pour son fils Rufius Rufianus, sa fille Rufia Pupa et pour sa fille Rufia Sacirata, morte à 22 ans, [élevé] ce tombeau avec une vigne et des murs, légué pour l'achèvement de la construction, [son entretien] et sa protection ainsi que pour un repas à donner le $14^{\mathrm{e}}$ jour des mois de 30 jours, la somme de ?? deniers à perpétuité. Ce tombeau est (dédié) sous l'ascia. Le monument (haec opera) ou son domaine (locus) ne passe pas à nos héritiers.

De l'inscription initiale ne subsistent que deux fragments, conservés l'un (fragment a) dans la maçonnerie d'angle nord du presbytère (placé à l'envers), l'autre (fragment b) comme support du bénitier dans l'église (malheureusement piqueté à une époque indéterminée).

Notre lecture s'établira ainsi : 
a) le monument

b) les modénatures

c) l'inscription

Fragment a)

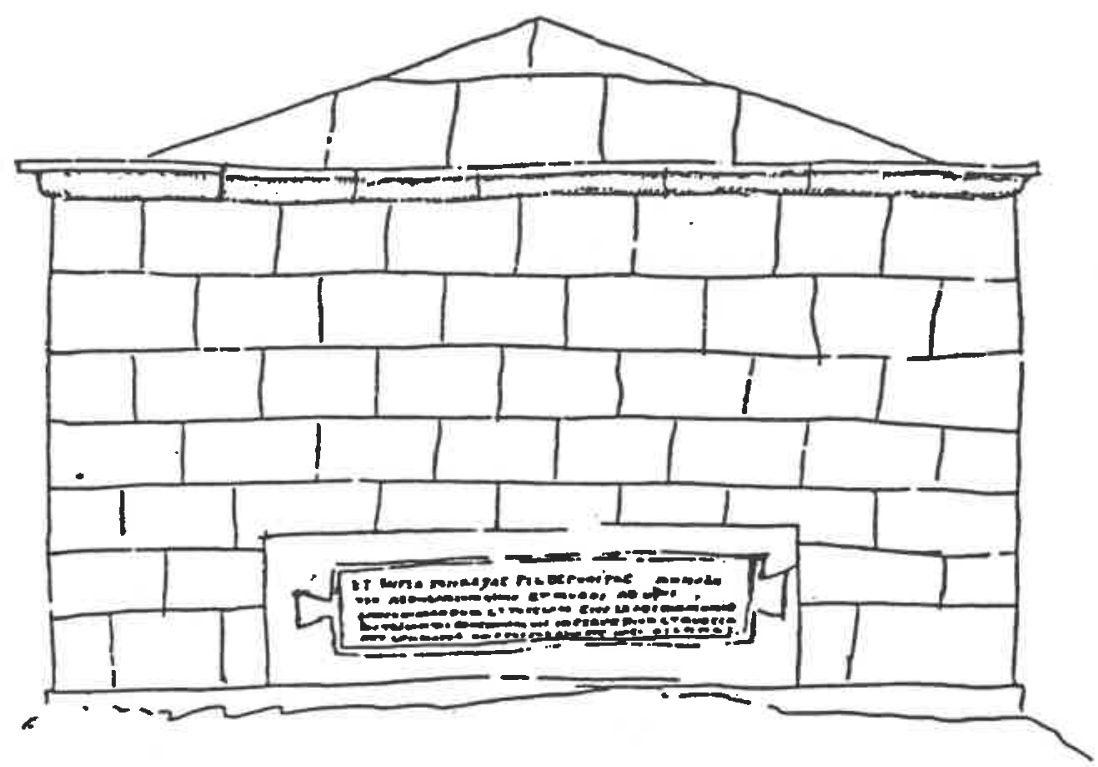

sans autre ornement qu'une corniche de cette façon:

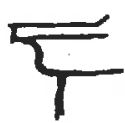

Le dessus de ce tombeau se terminoit en dos d'âne de cette façon:

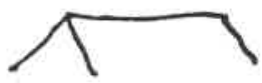

ET RVFIAE-SACIRATAE FILDEFNNCTAE ANNOR. XXIT. AEDICLAM.CVMVINLA.ET. MVRIS.AD OPVS CONSVMMANDVM-ET TVTELAM.EIVS.ET AD CENAM OMN BVSTRJCONTIS PONENAM XIF: IN PERPET SIOVTI. ERVDECA MF. COSVMATVR. HOC CIPVS SVB ASCIA EST HAECO.SLHN.G.S.

Fig. 2b. - Le Mausolée de M. Rufius Catullus dlaprès le dessin de C. de Veyle, Explication des Antiquités romaines..., p. 46. 
Partie supérieure :

[memoriae aeter

nae M. Rufius Catul]

LVS - CVRA[tor N(autarum). Rh(odanicorum)]

VIVVS•SIBI•E[t Ruf(io). Rufiano. f(ilio)]

RVF•PVPAE $\cdot F[i 1(i a e)]$

Partie inférieure :

[et. Rufia Sacirata def]VNCTAE - ANNOR

[XXII . aediclam cum uinea et mu]RIS - AD - OPVS

[consummandum et tutelam eius e]T $\cdot \mathrm{AD} \cdot \mathrm{CENAM} \cdot \mathrm{OMNI}$ [bus tricontis ponendam XII in perpet] SICVT - PETRVDECA

[meto consumatur hoc opus sub ascia est hae]C $\cdot \mathrm{O} \cdot \mathrm{S} \cdot \mathrm{L} \cdot \mathrm{H} \cdot \mathrm{N} \cdot \mathrm{S}$

On pourra présenter plusieurs remarques à propos de ce texte relativement long par rapport aux autres épitaphes découvertes dans la même région:

- l'invocation aux dieux Mânes est absente du texte, ce qui donne un critère de datation (voir plus loin);

— le mot «deniers» est présenté par son abréviation traditionnelle : le X barré horizontalement;

- après avoir soulevé de nombreuses difficultés de lecture, les deux termes tricontis et petrudecameto, désignant «trente» et «quatorzième», empruntés au vocabulaire celtique, ont été mis en relation avec le vocabulaire du calendrier de Coligny ${ }^{(8)}$. Rapprocher ces deux inscriptions (Coligny et Gélignieux) et éventuellement les fragments de calendrier trouvés au Lac d'Antre (Villars d'Héria, Jura), d'époque voisine, mais éloignés dans l'espace d'une centaine de kilomètres, permet de souligner l'existence d'une communauté culturelle dans laquelle la langue gauloise survivait, à l'état de langue vernaculaire ou de patois dans le massif du Jura méridional et, ce qui est plus intéressant, dans la langue des cérémonies religieuses ${ }^{(9)}$.

\section{LE PERSONNAGE ET SA FAMILLE}

Le tombeau a été bâti par Marcus Rufius Catullus à la mort de sa fille Sacirata pour abriter ses cendres et celles des autres membres de la cellule familiale. Une personne n'est toutefois pas mentionnée, il s'agit de l'épouse du fondateur, qui était peut-être disparue depuis longtemps et dont les cendres avaient pu être déposées ailleurs ${ }^{(10)}$, en l'absence d'un mausolée familial antérieur.

(8) Cr. J. Loth, L'inscription de Gélignieux et le prétendu Ligure ou Celtican du calendrier de Coligny: dans CRAI, 37, 1909, p. 16-28; petrudecameto = quarto decimo die : le quatorzième jour des mois de trente jours », peut-être en rapport avec le cycle défini par le comput de calendrier gaulois : «pour un repas de deux deniers (?) par tête à servir tous les mois de trente jours de telle sorte qu'il soit consommé le quatorzième jour».

(9) Ce que P.-M. Duval appelle du "gallo-romain campagnard»: intervention lors de la table-ronde CNRS sur Les inscriptions latines de Gaule Narbonnaise. Montpellier, 1983 (dactylographié), p. 50; voir également P.-M. Duval et G. Pinault, Recueil des inscriptions guuloises (RIG), III : Les calendriers (Coligny, Villars d'Héria), Paris, CNRS, 1986, XLVe supplément à Gallia, particulièrement carte 1, p. 2 et 433 .

(10) Mais où ? Le problème se pose indirectement de l'attachement du mari à sa femme. En effet, si cette dernière était đécédée antérieurement. il faut supposer que le tombeau familial aurait été bâti pour abriter en tout premier lieu ses cendres, et ensuite celles des autres membres de la famille. Il reste à supposer que cette personne avait eu une mort violente (noyade,) suivie d'une disparition du corps. 

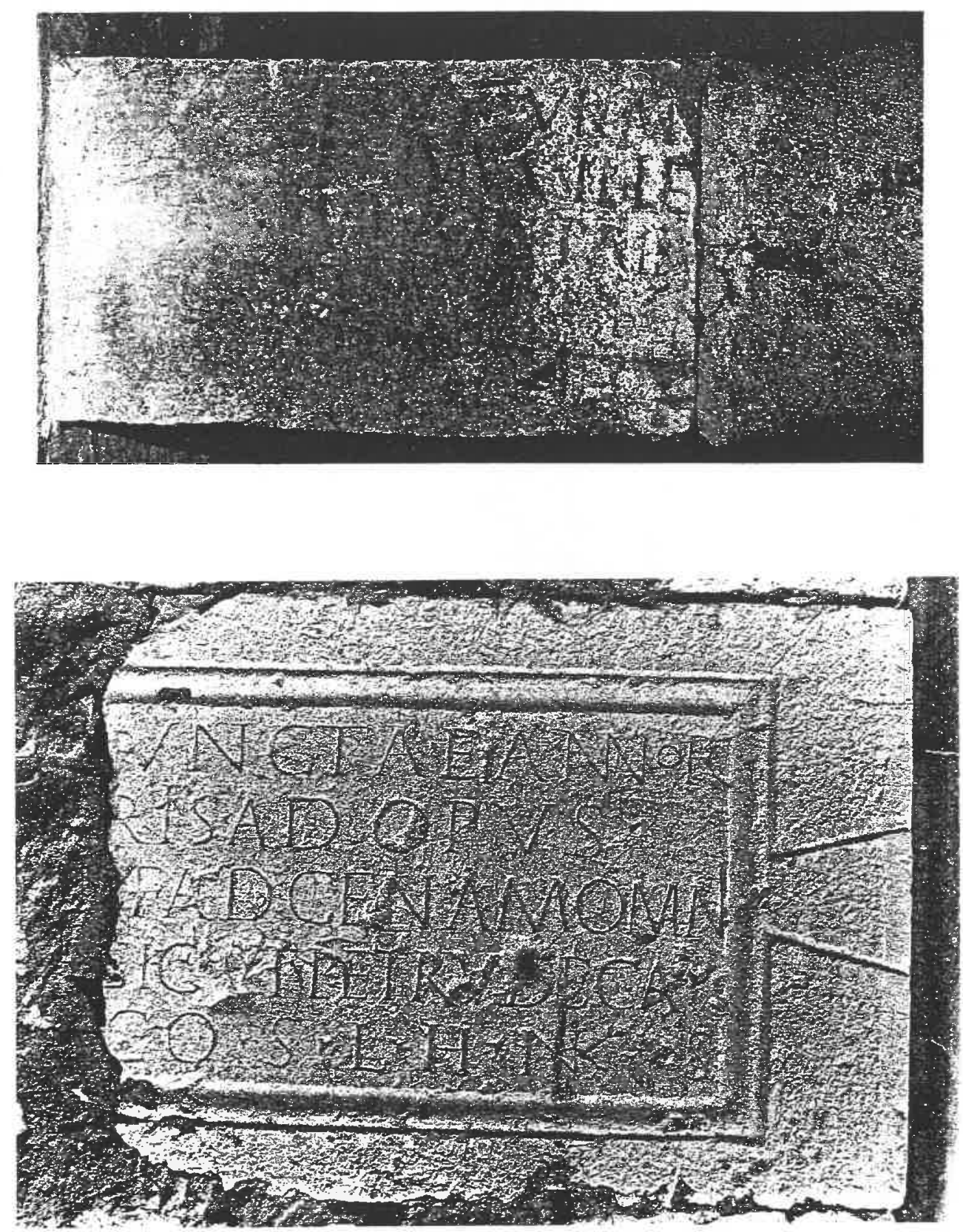

Fig. 3. - Les fragments conservés de l'inscription CIL XIII 2494. 


\section{Le nomen}

Les Rufii ou Ruffii portent un nomen d'origine latine bien attesté dans toute la Gaule et les Germanies ${ }^{(11)}$. Cependant, la région du Bas-Dauphiné et du Bugey se remarque par la forte densité des nomina qui semblent avoir fait souche dans quelques cas ${ }^{(12)}$. On les retrouve dans le territoire du premier diocèse de Belley; y a-t-il lieu d'y reconnaître également l'aire de colonisation romaine sur le territoire allobroge? (fig. 4).
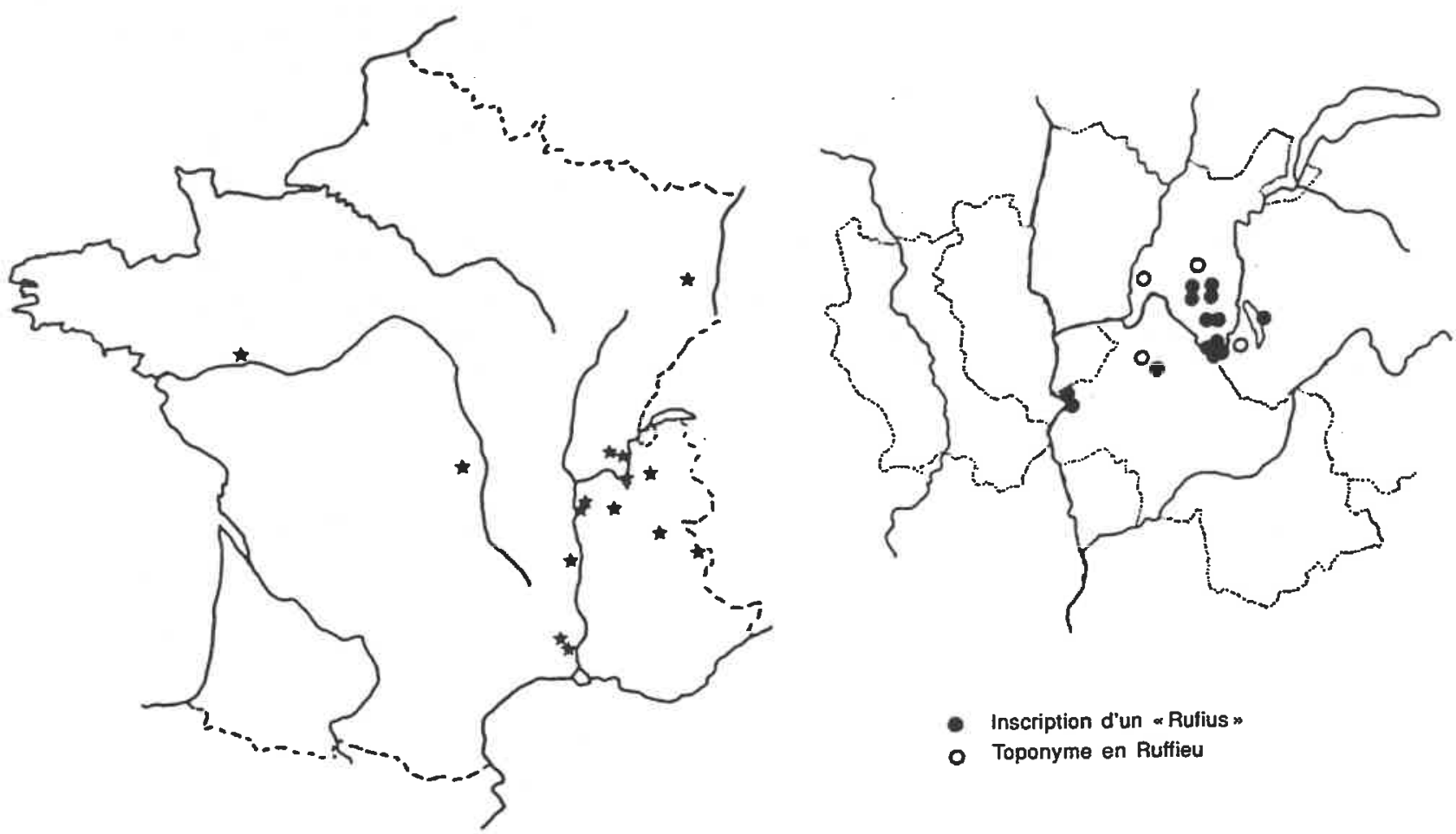

Fig. 4. - Le domaine d’implantation des Ruf(f)ii en Gaule et dans la région Rhône-Alpes.

(11) W. Schulze. Zur Geschichte Lateinischer Eigennamen, Berlin-Zurich-Dublin, (1 ${ }^{\text {re }}$ éd., Göttingen, 1904), $2^{\mathrm{e}}$ édition, 1966, p. 221 et 295. ClL XII, 133 (Alpes Poenines) : Rufius Archilius Sividius ; 1819 (Vienne) : L. Rufius Rufinus ; 2251a (Uriage) : M. Rufius Marcianus; 2359 (Saint-Savin): L. Rufius Cato; 2461 (Aix-les-Bains): Rufius Iulianus; 2724 (Saint-Poulet-de-Caisson): Q. Rufius Marcellinus : 3943 (Nîmes): M. Rufius Iustinus, Rufia Quartina, M. Rufius Maximinus; 5680,5 (Vienne : marque de potier): Rufius. CIL XIIl 1595 (Saint-Paulien): Rufius Rufinus: 2520 (Chazey-Bons): M. Rufius Cassiolus, Rufius Titulus; 2509 (Vieu-en-Valromey) : C. XIIl 1595 (Saint-Paulien) : Rufius Rufinus : 2520 (Chazey-Bons) : M. Rufiaus; 2541 (Vieu-en-Valromey) : C. Rufius Eutactus, C. Rufius Virilis: 3094 (Ayers) : C. Rufius ; 4704 (Rebenville) : S. Rufius Agricola; 6681,15 (Mayence) : Rufius Carinus; 7319a (Nied) : C. Rufius ; 7403 (Friedberg): Rufius Sennas; 8706 (Millingen) : Rufius Similis.

(12) Cf. CIL XII, 1819, 2461; CIL XIII, 2494, 2509, 2520, 2521, 2540, 2548; pour les toponymes en «Ruffieu»: cf. A. HoLDER, Alt-Celtischer Spraschatz. Leipzig, 3 vol., 1896 (réimpr. Graz, 1961), col. 1241, soit : Rufieu-en-Valromey, Ruffieu (cne de Proulieu, canton de Lagnieu. Ain). Rufieu (canton de Bourgoin), Rufieu (près des Eparres, cne de Nivollas-Vermelle), Ruffieu, (Savoie, près de Yenne). 


\section{Les cognomina}

Catullus : ce surnom est très répandu en Gaule et plus spécialement dans 1'Est ${ }^{(13)}$, où 1'orthographe avec un ou deux $L$ est variable dans la même région ${ }^{(14)}$. L'origine gauloise de ce surnom ne semble faire aucun doute ${ }^{(15)}$, de même que sa perduration au cours des siècles ${ }^{(16)}$. Marcus Rufius Catullus est peut-être déjà cité (par son cognomen uniquement) dans la référence «sub cura Catulli» de l'inscription de Groslée (P. Wuilleumier, ILTG, $\mathrm{n}^{\circ} 308$ ) comme exécuteur testamentaire de L. Varius Lucanus ${ }^{(17)}$.

Pupa: on le rencontre à plusieurs reprises dans la proche région, ainsi qu'à Lyon et dans la vallée du Rhin ${ }^{(18)}$. Mais la basse vallée du Rhône ${ }^{(19)}$ et la Gaule Cisalpine ${ }^{(20)}$ sont riches de ce cognomen, qui peut désigner tout simplement un sentiment d'affection envers la benjamine d'une famille.

Rufianus : est assez bien implanté localement, où trois inscriptions du Bas-Bugey le signalent (avec variante de redoublement du $F)^{(21)}$, encore que sa présence soit attestée aussi dans la vallée du Rhin et en Cisalpine, à Milan (22). On remarque que le cognomen tiré du nomen se retrouve sur trois cas du Bas-Bugey.

Sacirata: on le retrouve dans sa formulation classique de Sacrata, dans le département de la Côte-d'Or ${ }^{(23)}$. L'inscription de Gélignieux présente d'ailleurs peut-être une erreur de gravure (qui aurait fait rajouter une haste entre le $\mathrm{C}$ et le $\mathrm{R}$ pour donner une variante inconnue par ailleurs, à moins qu'il ne s'agisse d'une prononciation locale ou d'un phénomène lié à un surnom d'origine celtique).

Il est intéressant de noter, dans les tria nomina du dédicant, l'association du praenomen latin, du nomen d'origine latine et d'un cognomen typiquement gaulois. Ce pourrait être le reflet, à la fin $\mathrm{du} \mathrm{II}^{\mathrm{e}}$ siècle de notre ère ou au début du $\mathrm{III}^{\mathrm{e}}$, d'une mentalité gauloise dans un pays fortement romanisé, comme une ultime résistance à l'acculturation (?) (24).

(13) CIL XHI. 1140 (Poitiers), 2165 (Lyon). 2494 (Gélignieux), 5368 (Besançon), 5416-5418 (Mandeure), 8244 (Cologne), ILTG, 308 (Saint-Benoit, Ain).

(14) Catulius (ILTG 306, dédicace du fanum de Lhuis), Catulla (CIL XIII, 5706), Catulus (CIL XIII, 6801,8), Catullianus (CIL XIII, 4362. Metz). Catulianus (CIL XIII 2983, Sens). Catullinus (ClL XIII 2705, Autun; 3723, Trèves; 1691, Lyon; 6198, Brambach; 4517. Heidelberg...): Catulinus (CIL XIII. 6002, Saverne). Catullius (CIL XIII 4669, Bar-le-Duc), Catulus (CIL XIII 5706, Langres; 6801.8. Mayence...). En Narbonnaise. ses dérivés se retrouvent également à La Batie-Montsaléon (CIL XII, 1546), Vienne (CIL XII, 5680,3). Nîmes . (CIL XII, 3739. 3929, 4009), Arles (CIL XII, 763), Castellane (CIL XII, 65), Narbonne (CIL XII, 4569, 4691), Béziers (CIL XII. 4225). Grenoble (CIL XII. 227I), Le Bourget-du-Lac (CIL XII, 2435), Les Echelles (CIL XII, 2425).

(14) D. Ellis-Evass. Gaulish Persomal Nanes. a study on some continental Celtic Formations, Oxford, 1967, p. 171-175 et aussi W. SCHL'LZE. op. cit. p. 76; A. HOLDER. op. cir. col. 850.

(15) Des marques de potiers Catullus d'époque flavienne et antonine ont été trouvées sur des céramiques de Lezoux : cf. F. OSwALD, Index of Pollers Stamps on terra Sigillata "Samian Ware", East Bridgford, 1931, p. 66-67 et 369.

(17) Le lien entre les deux hommes serait alors fait par le côté professionnel et permettrait d'élucider les difficultés de lecture de la formule commubernium caruonenses de l"inscription de Groslée. qui serait non seulement une «confrérie ethnique à caractère religieux » (P. Wuilleumier), mais un collège funéraire lié aux Nautes.

(18) W. Scht'Lze. op. cit. p. 221 et 295: CIL XIII. 1985 (Lyon); 5976 (Kœnigshaven); 7412 (Grosskrotzenburg-Hanan). I. KAJA:TO. The Latin Cognomima. Helsinki. 1965. p. 300 (" cognomina relating to age», pour de très petits enfants).

(19) CIL XII. 1234 (Pupa. à Orange): 1391 (Pupa. à Vaison), 1640 (Pupus, à Die); 1659 (Pupus, à Die), 1678bis (Pupus, à Die), 1727 (Pupà à Crest).

(20) Pour le seul surnom Plupa. cf. CIL V. 4549, 5545, 2455, 2461, 3536, 5026, 5292, 5311, 5596, 5888, 5956, 6026, 7500, ... R. Chevallier faisait remarquer l'étroitesse des liens, soupçonnables par l'onomastique, entre le Rhône et la Celtique du Pô: R. CHEVAlLier el P. Dífolinet. Nouvelles inscriptions funéraires d'Anglefort, dans Le Bugey, 1981, p. 7-17 (part. p. 13).

(21) CIL XIII. 2486 (Ambléon) et 2548 (Vieu-en-Valromey).

(22) CIL XIII, 7565 (Wiesbaden) (Rufiana); CIL V, 5790 (Milan).

(23) CIL XIII. 5614 (Mirebeau), cf. Holder, op. cil. col. 1278-1279.

(24) A ce sujet. B. RÈMY, Les Allobroges, population et religion. études épigraphiques, thèse de doctorat de troisième cycle, Lyon, 1971, dactylographiée, p. 69-72. 
Les fonctions de M. Rufius Catullus: le personnage porte deux titres professionnels complémentaires : il est dit dans son épitaphe : naute du Rhône et curateur de la corporation.

Les nautes du Rhône avaient pour fonction l'acheminement des marchandises sur le cours du Rhône entre Lyon et Genève - ce qui nous rappelle que le transport fluvial était environ quatre fois moins cher que le transport terrestre ${ }^{(25)}$ - par répartition des tâches au sein même de leur

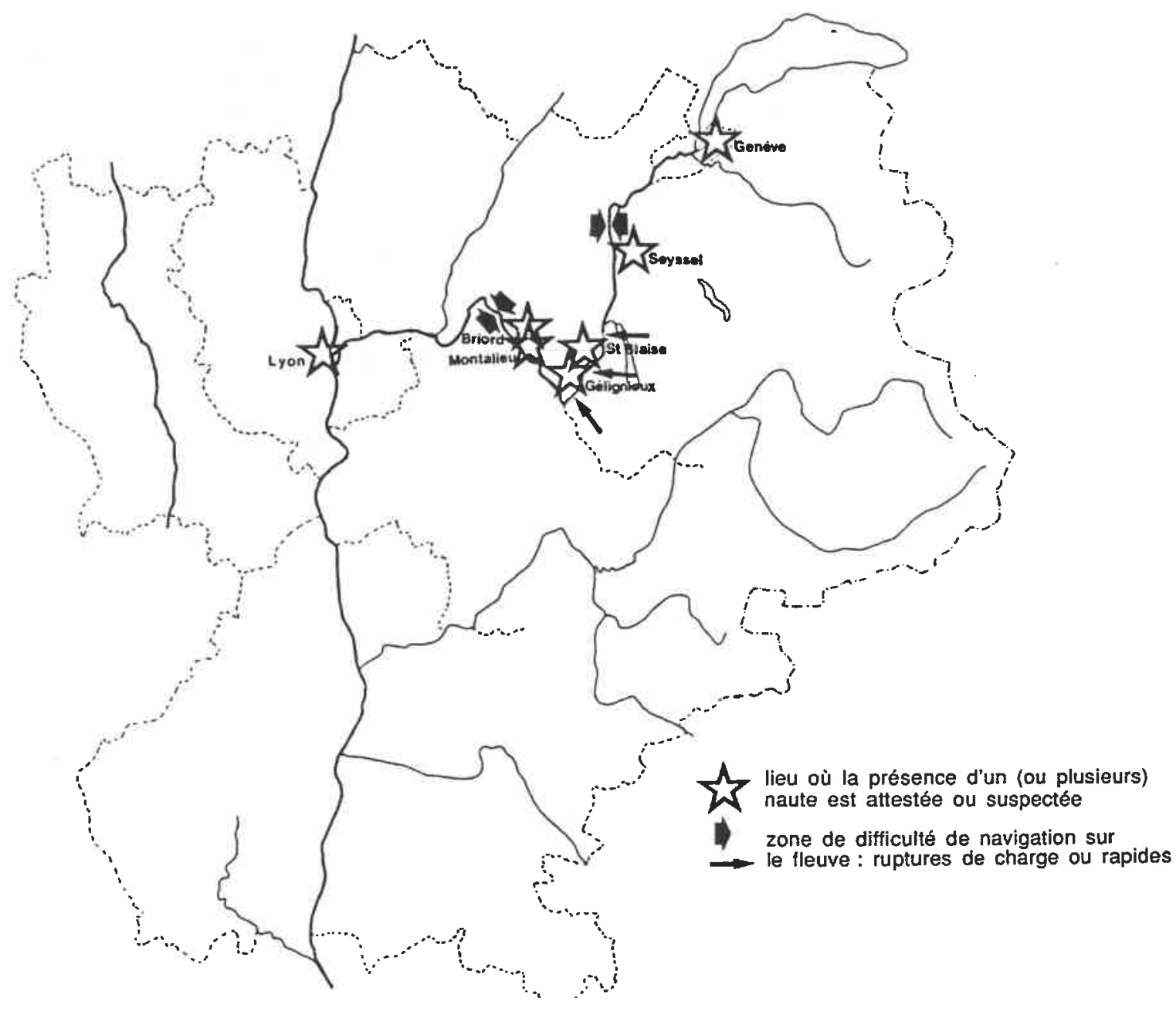

Fig. 5. - Les nautes du Haut-Rhône.

(25) D'après l'Edit du Maximum. de Dioclétien, 37. 74. Cf. A. Deman, Matériaux et réflexions pour servir à une étude du développement et du sous-développement dans les provinces de l'Empire romain, dans ANRW, II, 3, Berlin-New York, 1975, p. 3-97, particulièrement p. 7 note 6 . 
corporation (26). Ils possédaient plusieurs domaines le long de ce cours d'eau comme a pu nous le montrer une enquête sur le terrain ${ }^{(27)}$ (fig. 5).

Le personnage est dit curator de sa corporation, c'est-à-dire qu'il en a été l'administrateur au titre d'une charge sans doute élective, et par celle-ci, il a été le représentant de sa corporation professionnelle, dont, face à l'administration romaine, il gérait les finances et administrait les biens ${ }^{(28)}$.

\section{LE TOMBEAU DANS SON JARDIN ${ }^{(29)}$}

Le tombeau de Marcus Rufius Catullus se présentait comme un véritable cepotaphium dans la définition qu'en a donnée J.-J. Hatt ${ }^{(30)}$. Dans le texte de son épitaphe, l'auteur donne la formule suivante : aediclam cum vinea et muris. Comme dans l'inscription de Briord (CIL XIII 2464), il est question d'une vigne à destination funéraire. Dans ce cas précis, il s'agit d'une vigne liée au tombeau (peut-être une tonnelle), enclose dans l'enceinte de murs prévue dans le testament; par ce biais, le tombeau prend un caractère de cepotaphium, avec des installations pour les convives au moment du banquet anniversaire.

Plusieurs caractéristiques apparaissent dans le soin donné par le fondateur à la préservation de son tombeau : certaines font référence au droit funéraire et seront développées plus loin, d'autres sont de simples précautions. Il faut d'abord noter de la part de M. Rufius Catullus le souci de l'isolement de la tombe, dans un enclos entouré de murs ou de murets, mais aussi un souci d'établir le tombeau dans la longue durée, grâce à la prescription d'une fondation funéraire dont le but est double : l'entretien et l'achèvement de la construction du tombeau ad opus consummandum et tutelam eius et le repas à offrir tous les «quatorzième jour» des mois de «trente jours», soit 6 fois par an et ad cenam omnibus tricontis ponendam denariis II in perpet(ua) sicut petrudecameto consumatur aux membres d'un groupe humain non précisé dans l'inscription mais qui devait aller de soi lors de la création du tombeau et pour un montant par tête de " $x$ » deniers (la somme indiquée a été corrodée sur la pierre) (31). On a donc envisagé qu'il pouvait s'agir des membres de la corporation des nautes dont le fondateur du tombeau avait été le curateur, bien qu'il puisse être question d'autres collectivités, comme celle formée par un collège funéraire dont l'auteur aurait eu la charge à une certaine époque (si l'on envisage un lien entre M. Rufius Catullus et l'inscription de Groslée ILTG 306).

(26) Nautes du Rhône, nautes du Rhône et de la Saône, nautes de la Saône et de la Loire, nautes du lac Léman, ratiarii superiores. Cf. P. Wuillelimier. Lyon, mérropole des Gaules. Paris, 1953, p. 50 «les différences d'appellation peuvent s'expliquer par la division d'une seule et même société en branches multiples»; dans le même sens, J. RouGÉ, Aspects économiques du Lyon antique, dans Actes du colloque sur les Martyrs de Lvon (177). Paris, CNRS, 1978, p. 47-63, particulièrement p. 53.

(27) Cf. Par exemple CIL XII 2438 (= All.Mer et Terrebasse, Inscriptions antiques et du Moyen-Age de Vienne en Dauphiné, Vienne, 1886, t. Il, n" 155): M. Marujno Mal---] / lurihina[--1/ Rhodo V[---]/ dint Corpor[---] / ri[--]itimo Val[---] / ior hi patrono Maruinus / Marcellus filius patri / pientissimo p(onendum) $\mathrm{c}$ (urauit) et $\mathrm{s}(\mathrm{ub}) \mathrm{a}(\mathrm{scia}) \mathrm{d}(\mathrm{edicauit})$, que l'on doit rapporter à Virignin Ain. rive droite du Rhône, plutốt qu'à la proche région de Yenne - Savoie, rive gauche du Rhône. La lecture de ce texte fait problème Auguste Allmer lui-même considérait qư avant de hasarder une lecture complète, il faudrait retrouver la pierse, déjà usée et corrodée.

(28) J.-P. WaltziNG, Etudes historiques sur les corporations professionnelles chez les Romains, depuis les origines jusqu'à la chure de l'Empire d'Occident, Louvain, + vol., 1895-1900, réimpr. Rome, 1968, vol. I, p. 408-409.

(29) Nous avons ailleurs présenté les nombreux points communs de ce tombeau avec celui décrit dans le fameux «testament du Lingon” (CIL XIII $5708=$ Dessau. ILS. $\pi^{\circ} 8379$ : A. BLISsox, Le tombeau du Lingon, Etude du cadre architectural et archéologique, dans Y. Le Bohec et A. Busson (éditeurs), Le Testamen du Lingon. Actes de la journée d'études du 16 mai 1990, Lyon, collection du CERGR, n"9, 1991. p. 63-72.

(30) J.-J. HaTt, La tombe gallo-romaine, Paris, 1951, 2édition 1986; p. 71-72; voir également P. GrimAl, Les Jardins romains, Paris, Fayard, $3^{e}$ édition, 1984. particulièrement p. 59-60.

(31) Cf. aussi : Y. DE KISCH. Tarifs de donation en Gaule romaine, dans Ktéma 4, 1979, p. 259-280; J.-F. DRINKWATER, Gallic Personnal Wealth, dans Chiron. 9, 1979, p. 237-242; cf. aussi B. LAUM. Stiftungen in der griechischen und römischen Antike, Leipzig, 1914 (CIL XIII 2494=n" 94, p. 186). Comme l'a bien mis en évidence M. Le Glay, ił ne servirait à rien de tenter d'épiloguer sur le rythme même des cérémonies funéraires (M. LE Glay, Notes sur le Testament du Lingon, dans Le Testament du Lingon Actes de la journée d'étude du 16 mai, 1991, p. 59). 
L'enceinte du monument funéraire était marquée au sol par un mur ou muret (aediclam cum vinea et muris), dont aucune trace n'a perduré, sauf peut-être le cippe-autel encore conservé aujourd'hui à proximité de l'emplacement du tombeau ${ }^{(32)}$ (fig. 6).

\section{LE MONUMENT FUNÉRAIRE}

Une remarque préliminaire s'impose : le monument n'était pas achevé lors du décès de Rufia Sacirata (d'après les termes mêmes de l'inscription : et ad opus consummandum). Il devait comporter, autour du tombeau proprement dit, une clôture formée d'un mur et une vigne. Il s'agissait donc d'une véritable enceinte sacrée bordée de murs ou de murets de pierre (comme une projection du domaine agricole idéal).

La description de Claude de Veyle est relativement précise : dans son état au début du XVIII siècle, le monument est décrit ainsi : "Voici un autre tombeau qu'on voyoit à Génilieu (sic), paroisse sur le bord du Rhône près la pointe qu'il forme à Cordon à deux ou trois lieues de Belley en descendant cette rivière (...). J'en puis donner la figure parce que $\mathrm{j}$ 'en ay vu les fondements et la corniche. C'étoit une grosse masse de pierre de 13 ou 14 pieds en carré et de huit pieds de haut surmonté d'une grosse pierre qui finissoit en pointe et formoit un fronton triangulaire de deux côtés, sous lequel étoit la corniche qui couronnoit l'œuvre. Ce tombeau étoit bâti de forts gros morceaux de pierre qui devoient lui permettre une durée éternelle. Beaucoup de fer et de plomb en faisoient les liaisons. On entroit dans ce tombeau par une ouverture quarrée de 4 ou 5 pieds et plusieurs personnes pouvoient se tenir dans ce petit édifice fort à leur aise».

Il apparaît très nettement que ce que de Veyle décrit avec une précision méritoire (le monument avait alors été détruit depuis près de quinze ans) ne forme que la partie inférieure du tombeau, le

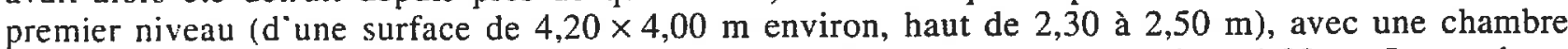
funéraire fermée par une porte dont la hauteur sous le linteau était d'environ $1,30 \mathrm{~m}$. Le tombeau avait environ huit pieds de hauteur sous la corniche moulurée qui délimitait la base proprement dite, dans laquelle était ménagée une chambre funéraire, haute de 7 pieds environ, dans laquelle on pénétrait par une porte haute de 4 pieds («depuis la corniche au rez-de-chaussée en dehors (croquis), mais au dedans, il étoit plus petit d'un bon pied. On y entroit par une porte d'environ quatre pieds de haut. Le dessus de ce tombeau se terminoit en dos d'âne de cette façon (croquis), sans autre ornement qu'une corniche de cette façon» de Veyle (croquis)). Le dessus du tombeau se terminait-il en toit à double pente comme semble l'indiquer de Veyle ? Malgré la précision de la description de de Veyle. il est peu probable que le tombeau ait eu une couverture aussi simple qu'un toit à double pente: celle-ci est sans doute plus vraisemblablement le résultat de la désagrégation de niveaux supérieurs plus fragiles. disparus, pour lesquels on est réduit à des conjectures dont nous donnons quelques éléments de comparaison. En effet, il ne fait aucun doute que la situation du monument lui donnait un rôle important dans l'aide à la navigation, à la manière d'un «amer». Il est donc

(32) Cf. notre commentaire : Le mausolée de Marcus Rufius Catullus, curateur des nautes du Rhône, à Gélignieux (Ain), dans Communications au $112^{*}$ Congrès National des Sociétés Savantes, Lyon, 1987. 

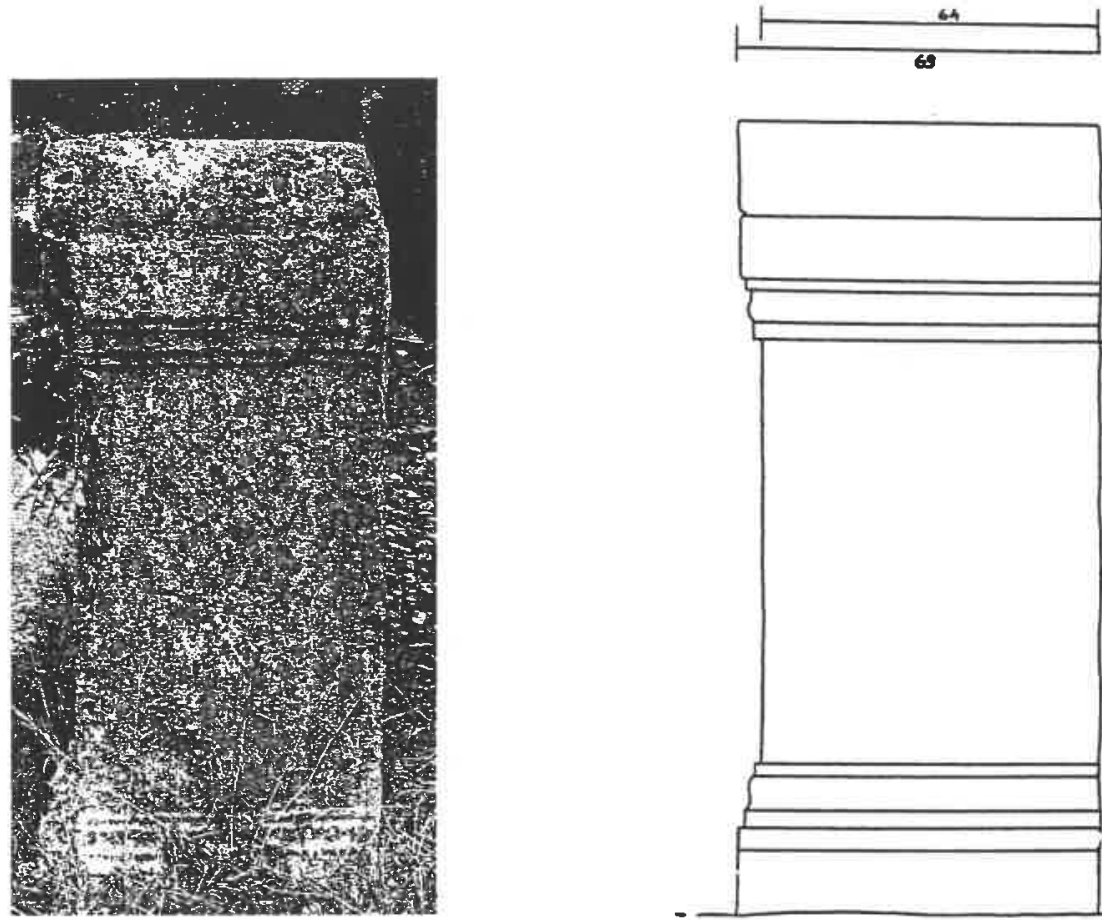

6
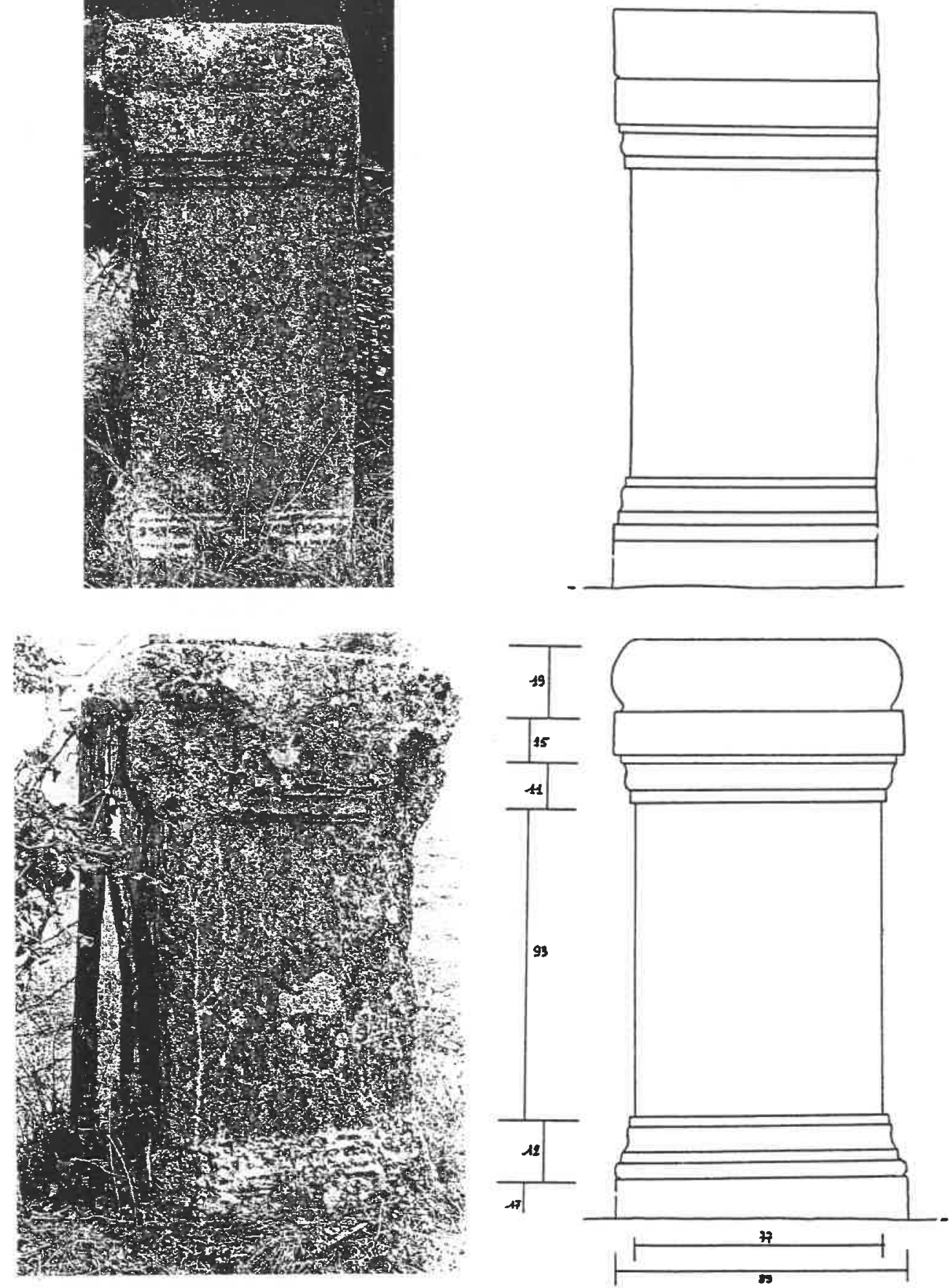

Fig. 6. - Le cippe-autel anépigraphe, le long du chemin de desserte. 
vraisemblable que le monument ait comporté plusieurs étages superposés, dans un souci de hauteur (préoccupation funéraire, mais également utilitaire), comme on le remarque sur les grands mausolées du monde romain encore conservés ${ }^{(33)}$.

\section{LE DROIT DU TOMBEAU}

La première des prescriptions est l'apposition sur le mur extérieur du tombeau des volontés du défunt; elle forme la garantie que les volontés émises par le défunt dans son testament seront respectées à tout jamais.

La protection du tombeau : elle est de deux ordres.

On trouve tout d'abord à la première ligne la mention de la memoria aeterna ${ }^{(34)}$ et à la dernière ligne la protection de l'ascia (hoc opus sub ascia est) qui soumet le monument à un régime particulier ${ }^{(35)}$; la seconde est la «clause d'exclusivité»: le tombeau est placé sous la protection juridique de la formule HOSLHNS (haec opera siue locus heredem non sequetur) qui s'apparente à des formules plus classiques comme HMHNS (hoc monumentum heredem non sequetur), ou HMHENS (hoc monumentum heredem exterum non sequetur) que l'on rencontre fréquemment et qui restreint aux personnes citées la destination du tombeau familial ${ }^{(36)}$. Le tombeau est donc un monument «exclusif», au sens où la clause d'exclusivité sert à éliminer les héritiers testamentaires extranei, «et constitue la clause la plus énergique du caractère familial d'une tombe, de sa destination exclusive aux agnats, affranchis...» ${ }^{(37)}$.

\section{LA VIGNE}

L'inscription donne un témoignage précieux sur les cultures locales. Sans oublier qu'il s'agit d'une culture à destination funéraire. nous pouvons rapprocher ces renseignements d'autres portés par des inscriptions proches géographiquement. En Gaule, six inscriptions mentionnent l'existence de jardins funéraires ${ }^{13 \mathrm{w}}$ dont trois avec une vigne (Briord, Gélignieux, Die), et une inscription men-

(33) Lexaltation de la hauteur semhle swrité une préoccupation constante des fondateurs de grands mausolées familiaux ou personnels: pour l'A rriqu. les cites lavii à Cillium (CIL VIII, 212-213), dans L'Afrique dans l'Occident Romain fer s. as: J.C. - M" wele ap. J.C. Actes du colloqui de Rome, déc. 1987 (1990), p. 49-61; J.-J. Hatt a également souligné

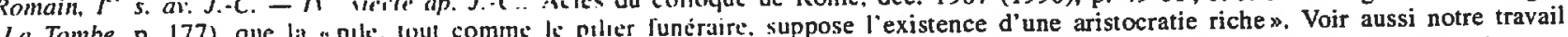
sur le tombeau du Lingon. deja cilé plus haul. Le umbeau est un repère pour les voyageurs (cf. le tombeau de Gordien III, dans Ammien Marcellix, Histoires, XXIll. 5,7 icid. J. Funlanne. Collection des Liniversités de France, 1977).

(34) La mention de la memoria aetermas. i slle seule, donne au tombeau une dimension religieuse.

(35) F. DE Visscher. La afrul des tombeas remesins, Milan. 1963, p. 281.

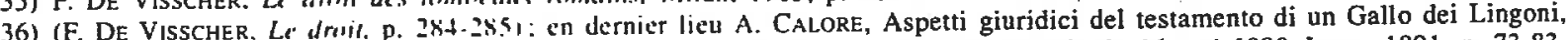

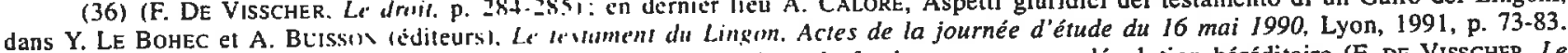
La formule met en avant le caracterce familial du lumbeau. imposé par le fondateur, avec non-dévolution héréditaire (F. DE VissCHER, Le droit, part. p. 93-122). Commc corrollaire à celte inlerdiction. une "peine sépulcrale» (multa) avait sans doute été prévue.

(37) F. DE VISSCHER. Le' drrit. part. p. $28+285$

(38) CIL XII 1657, à Dic: 3657 à Nimé: CIL XIII. 1072 à Saintes, 2464-65 à Briord, 2494 à Gélignieux, 5708 à Langres. 

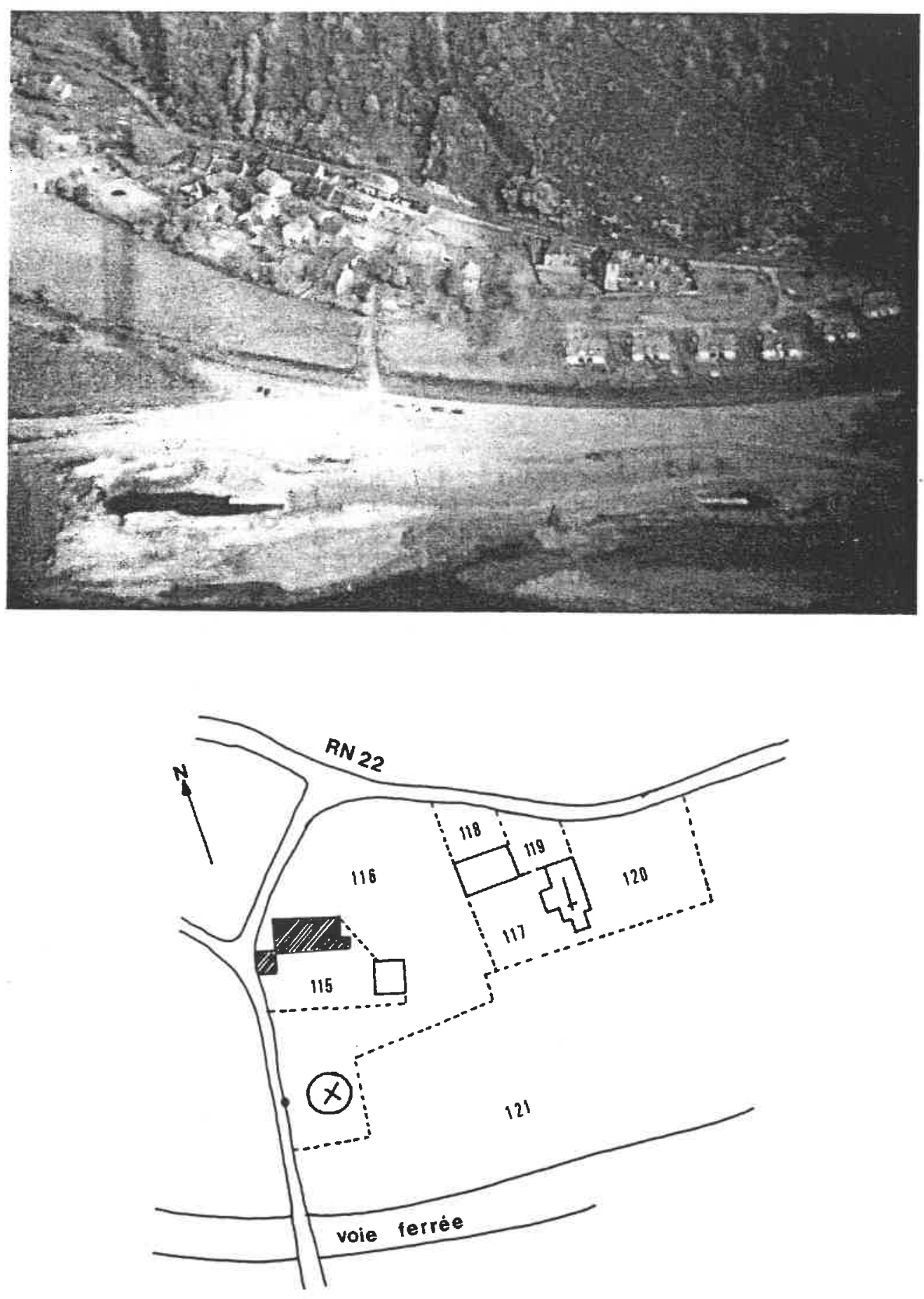

Fig. 7. - Plan et photographie aérienne du site. 
tionne la donation d'une vigne et d'un bois ${ }^{(39)}$ dans un contexte «public ${ }^{(40)}$. Ni le nombre, ni la répartition géographique, ni la destination de ces plantations ne sont le reflet de la situation de la viticulture en Gaule romaine ${ }^{(41)}$. Trois de ces inscriptions mentionnent des vignes situées dans la région lyonnaise, plus précisément dans ce que l'on nomme le Bas-Bugey et le Petit Bugey savoyard, sur des terroirs dont les caractères et l'exposition ont, notamment depuis le XVII ${ }^{e}$ siècle, favorisé le développement d'un vignoble de qualité. Sont-elles le témoin de la réalité régionale ou bien le contexte strictement funéraire de la plantation dans deux des cas a-t-il joué le rôle d'une culture «expérimentale»? (fig. 8).

Le vocabulaire comparé de ces inscriptions montre à l'évidence deux développements de la culture de la vigne :

- à Gélignieux, la plantation de vigne semble limitée à l'intérieur de l'enclos funéraire (aediclam cum uinea et muris...) et ne semble pas avoir été étendue à l'extérieur;

- à Briord, comme à Aix-les-Bains et à Die, la vigne semble avoir fait l'objet d'une culture en terrain ouvert, dont une certaine surface (un arpent $=4221 \mathrm{~m}^{2}$ ) aurait été réservée à la célébration des parentalia (... ad parentalia celebranda uinae arepennis... $)^{(42)}$. On pourrait donc déduire logiquement que la vigne, notamment dans le cas de l'inscription de Briord, n'était pas liée topographiquement au tombeau, mais que, située sur un coteau voisin, elle était entourée d'autres parcelles cultivées de la même manière; seul le revenu de cette parcelle aurait fait l'objet d'une destination funéraire par testament du propriétaire (cuius reditu...). Le cas de l'inscription d'Aix-les-Bains explicite encore cet exemple, puisqu'il s'agit du don à la communauté d'un bois et de sa vigne, autrement dit d'un ensemble de parcelles cultivées ou tout au moins entretenues, mais sans lien topographique avec leur destination.

Nous aurions donc, dans le premier cas, à Gélignieux, une vigne symbolique, de rendement juste suffisant pour l'entretien de la fondation funéraire, peut-être plantée en tonnelle ou en treille, et dans le cas de Briord, une parcelle appartenant à un vignoble de grandes dimensions. Seule intervient dans le calcul de la superficie de vigne de Gélignieux, la notion de calcul du rendement imposé dès l'origine à cette vigne; le vocabulaire de l'inscription est trop imprécis, et les données de l'archéologie sont inexistantes pour le muret de clôture de l'enclos.

\section{LE MATÉRIAU DE CONSTRUCTION}

N'ayant pu localiser tous les blocs qui composaient l'édifice, nous avons dû nous contenter de l'examen des trois blocs que nous connaissons (les deux fragments de l'inscription et le cippe) ainsi que de quelques blocs atypiques mais provenant sans doute du même monument, pour déterminer le matériau utilisé.

(39) L'existence d'une culture de la vigne porte en elle l'obligation de cultures connexes, telle l'osier, nécessaire pour fournir les échalas et les liens. Vigne et bois étaient ainsi étroitement liés, comme le souligne l'inscription d'Aix-les-Bains : lucus cum sua uinea. C'est encore vrai aujourd'hui, où les vignes sont toujours bordées de haies de saules ou d'osiers, quand parcelles viticoles et sylvicoles ne sont pas tout simplement juxıaposées, comme en Bas-Bugey. Cf. J. A.NDRÉ, La vigne et la forêt, sur un proverbe latin (CiCÉRoN, De

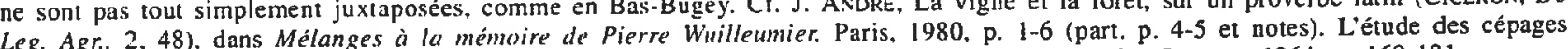
est développée par J. ANDré et L. Levadolג. La vigne el le vin des Allobroges, dans Journal des Savants, 1964, p. 169-181.

(40) A Aix-les-Bains. AE. 1934, n 165 .

(41) R. Dion, Histoire de la vigne et du vin en France, des origines au XIX' siècle, Paris, 1959, p. 71 et 135-136; E. DESJARDINS, Géographie de la Gaule, Paris. 1876, t. 1, p. 448 ; J.-J. HATT, op. cil.. p. 71.

(42) R. LEBEAL, La vie rurale dans les monagnes du Jura méridional, Lyon, 1955 (IER), p. 411-429. 


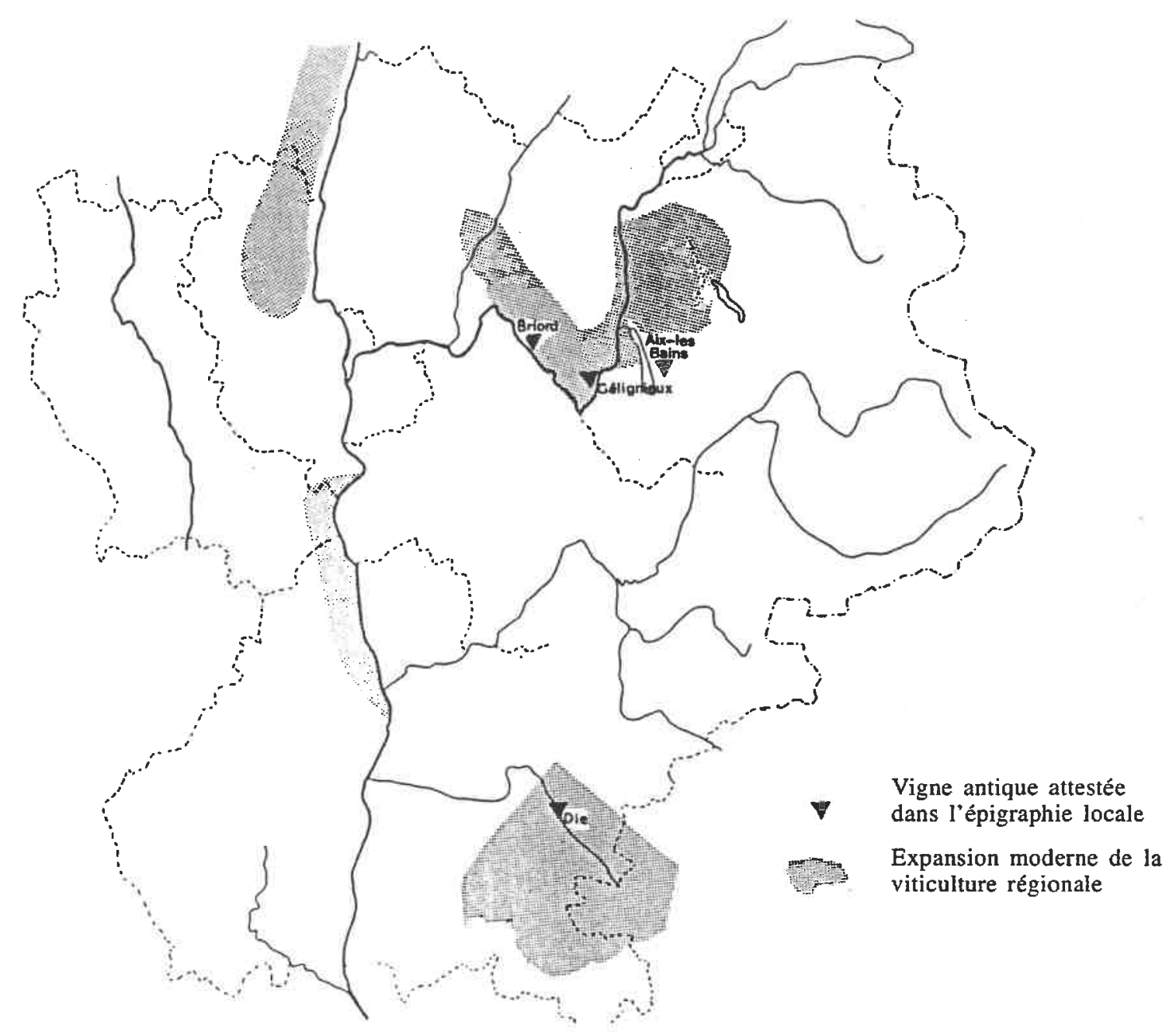

Fig. 8. - Vigne funéraire et publique.

Il s’agit de calcaire de l'ère secondaire, du Portlandien, dit également «choin de Fay» ou «marbre bâtard»dont les lieux d'exploitation sont, pour les plus proches du site, distants de quelques centaines de mètres ${ }^{43^{3}}$. Le matériau réagit à l'exposition aux intempéries en prenant une couleur blanche brillante, proche du marbre. qui devait avoir une grande importance dans la présentation du monument antique et des huts que lui avait assignés son fondateur.

(43) Cf. G. Mazenot, Les rewurces minérals de la régiun Isunnaise. dans Efudes Rhodaniennes, XII, 1936, p. 123-257 (parti-

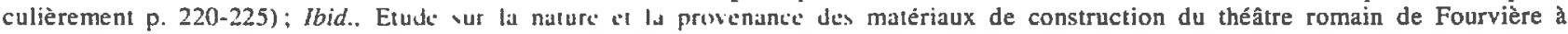
Lyon, dans Etudes Rhodaniennes, XVI. 1940-1941. p 140.172 (part. p. 166). Les carrières de pierre de Fay prenaient la forme de petites découvertes très difficiles à localiser attuellement. dupernées dans le secteur de la source «Merveille» sur la commune de Peyrieu et également le long des pentes de la muntiggne : cl. H SWAY-Gl-ERraz. Les matériaux calcaires dans l'art funéraire à Lyon, dans Gallia, 47, 1990, p. 135-144. Voir également J. RoťGltk. Proncinante du matériel lithique des monuments antiques de la région d'Aoste (Isère), dans Colloque international sur les rewrurces minerules. Grenoble. 1983. p. 349-360, particulièrement p. 355. 


\section{LA DATATION DU TOMBEAU}

Seule la formule funéraire peut être utilisée dans cette étude : par rapport aux inscriptions funéraires de la région, dont peu ont une aussi grande longueur, on remarque l'absence de la dédicace aux dieux Mânes, ce qui est un signe de postériorité (44). On daterait donc cette inscription de la seconde moitié du $\mathrm{II}^{\mathrm{e}}$ siècle, voire du début $\mathrm{du} \mathrm{III}^{\mathrm{e}}$ siècle de notre ère.

\section{ANNEXE}

\section{PROPOSITIONS DE RESTITUTION DE LA FORME DU MAUSOLÉE (fig. 9).}

Il est bien hasardeux de se lancer dans une telle entreprise, lorsqu'on ne possède que des éléments concernant le socle seul d'un monument. Pourtant, la recherche en la matière a fait de tels progrès qu'il est possible de proposer des pistes de travail dans ce sens. Nous avons donc cherché à créer des séries à partir de mausolées découverts, pour le plus grand nombre d'entre eux, dans l'Occident romain, et dont la base présente des dimensions proches de celles données par de Veyle pour le tombeau de Gélignieux.

Des comparaisons multiples sont possibles. On retrouvera des similitudes avec le mausolée dans : AAVV, Le mausolée de Cucuron (Vaucluse), dans Gallia, 47, 1990, p. 145-202; Y. BuRNAND, A propos des monuments funéraires de Cornillon, dans Provence Historique, 17, 1967, p. 394-395; G. MANSUELLI, Les monuments commémoratifs romains de la vallée du Pô, dans Monuments Piot, 53, 1963, p. 19-93; etc., mais plus particulièrement, dans les séries mieux définies de :

\section{Monuments à colonnade et statues:}

Y. BLRNAND, Domitii Aquenses, une famille de chevaliers romains de la région d'Aix-en-Provence, mausolée et domaine. Paris, 1975, 5 e suppl. à la Revue Archéologique de Narbonnaise; P. BRIDEL, Le mausolée de Wavre, étude des fragments architecturaux du Musée archéologique de Neuchâtel, dans $J b S G U, 59,1976$, p. 193-201.

\section{Monuments en forme de temple à colonnes de façade:}

A. AlLMER et P. DiSSARD, Trion. Antiquités découvertes en 1885,1886 et antérieurement, au quartier de Lyon dit Triom. Lyon, 1887-1888, p. 270-290: Id., Musée de Lvon, Inscriptions antiques, II, Lyon, 1889, p. 321 ; E. DESFORGES, G. et P.-F. FOURNIER. J.-J. HATT, Nouvelles recherches sur les origines de Clermont-Ferrand, Clermont, 1970. p. 250-25i. fig. 3 (mausolée du lieu-dit Pont-de-Naud); N. FERCHIOU, Un petit monument de Mididi : temple ou mausolée, dans Antiquités Africaines. 21,1985, p. $159-172$ (datable de la même époque que notre tombeau).

\section{Monuments en forme de pile ou de tour:}

H. KäHLER. Die Rheinischen Pfeilerdenkmäler. dans Bonner Jahrbucher, 139, 1934, p. 159 sq.; E. WILL, La tour funéraire de Palmyre. dans Siria, 26. 1949, p. 87-116; Id., La tour funéraire de la Syrie et les monuments apparentés, dans Siria, 26, 1949, p. 258-312 (c'est sans doute au Proche Orient qu'il faut rechercher l'origine de ce type usité en Gaule).

(44) II s'agirait donc d'une inscription relativement tardive. d'après les chronologies des formulaires funéraires : cf. par exemple J.-J. Hatt, op. cit., p. 12-19, affinée pour notre région par les études de A. AUdiN et Y. BuRnAND, Chronologie des épitaphes romaines de Lyon. dans REA. LXI. 3-4, 1959, p. 320-335 et tableaux et de Y. BURNAND, Chronologie des épitaphes romaines de Vienne (Isère), dans REA. LXI11, 3-4, 1961, p. 291-306 et tableaux. En dernier lieu, Y. BuRNAND, La datation des épitaphes romaines de Lyon, remarques complémentiaires, dans Inscriptions latines de Goule lyonnaise. Actes de la Table ronde de novembre 1990, Lyon, 1992, coll. du CERGR, $n^{\circ}$ 10. p. $21-27$. 


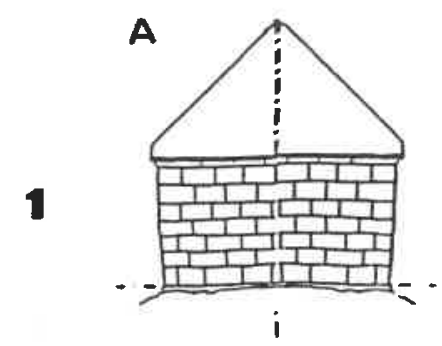

B
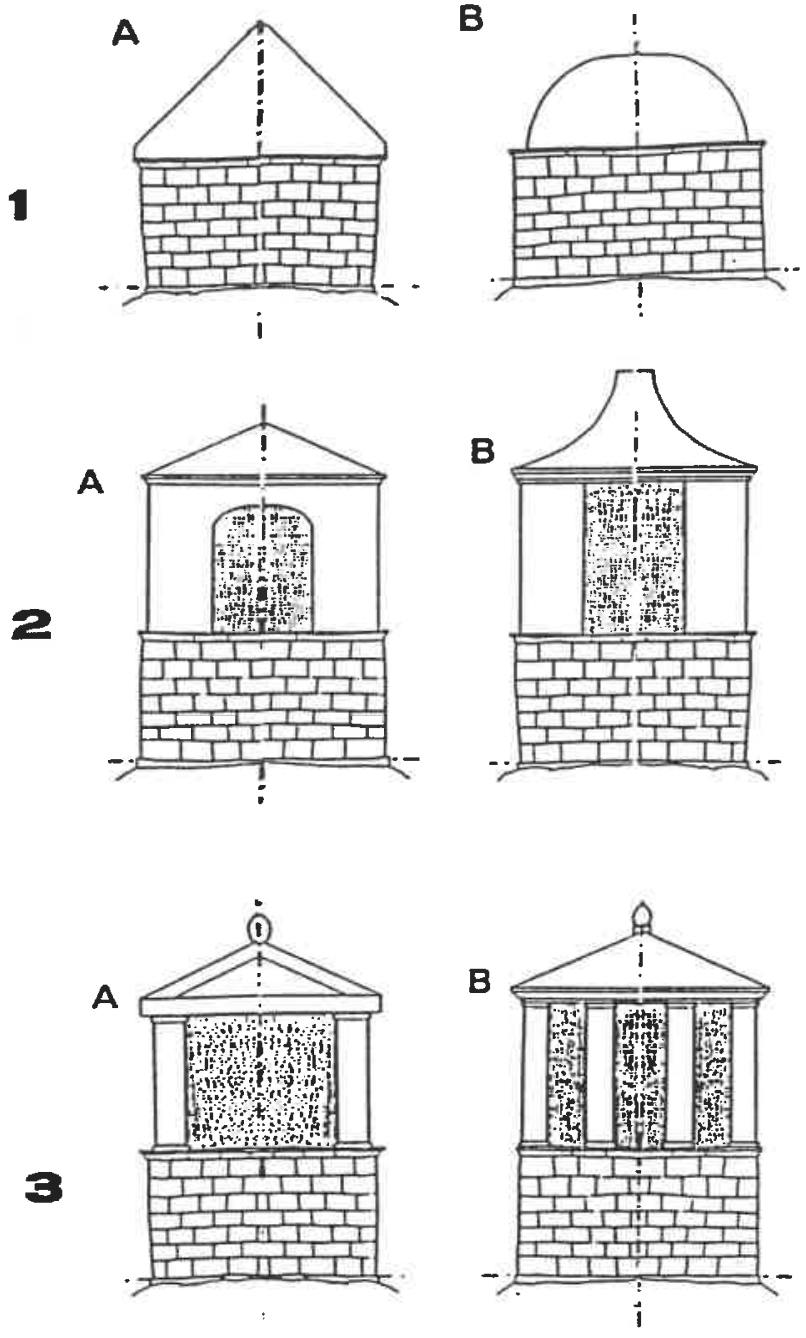

dans la vignette: mausolée de Gélignieux (d'après le dessin de C. De Veyte)

1. Mausolées d'Algérie (d'après S. Gsell) a: Akbou de l'Oued Sahe

(Gsell, fig.94 et p.62)

b: Mons (au nord-ouest de Sétif) (Gsell, $n^{\circ} 34, p .78$ )

2. Mausolées de Syrie ( $d$ 'après Cumont) a: Assai

3: Mausolées en forme de temple (Gaule). a: Warre (Suisse) d'après Bridel.

b: Lyon, tombe des Acceptii (d'aptès Allmer et Dissard)

4: Mausolées-tours

a: Villelongue d'Aude

(d'apres Barruol)

b: Lyon, tombe de Trion

(d'apres Kähler).
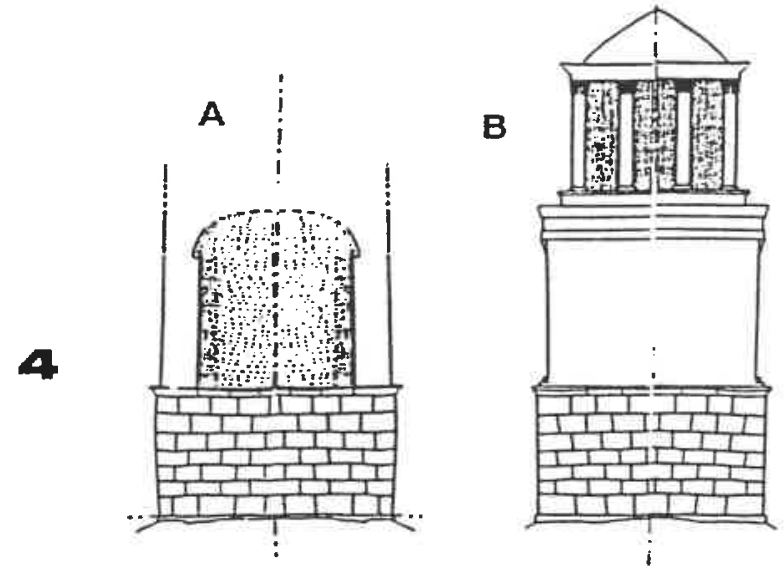

Fig. 9. - Mausolées : comparaisons. 
